\title{
Software design for the TAIGA-IACT telescope pointing and control system
}

D. Zhurov ${ }^{* 1,2}$, O. Gress ${ }^{1}$, I. Astapov ${ }^{3}$, P. Bezyazeekov ${ }^{1}$, V. Boreyko ${ }^{4}$, A. Borodin ${ }^{4}$, M. Brueckner ${ }^{5}$, N. Budnev ${ }^{1}$, A. Chiavassa ${ }^{6}$, A. Dyachok ${ }^{1}$, O. Fedorov ${ }^{1}$, A. Gafarov ${ }^{1}$, A. Garmash ${ }^{7}$, N. Gorbunov ${ }^{4,8}$, V. Grebenyuk ${ }^{4,8}$, T. Gress ${ }^{1}$, A. Grinyuk ${ }^{4}$, O. Grishin ${ }^{1}$, D. Horns ${ }^{9}$, A. Ivanova ${ }^{1}$, N. Kalmykov ${ }^{10}$, Y. Kazarina ${ }^{1}$, V. Kindin ${ }^{3}$, P. Kirilenko ${ }^{7}$, S. Kiryuhin ${ }^{1}$, R. Kokoulin ${ }^{3}$, K. Kompaniets ${ }^{3}$, E. Korosteleva ${ }^{10}$, V. Kozhin ${ }^{10}$, E. Kravchenko ${ }^{7,11}$, M. Kunnas ${ }^{9}$, L. Kuzmichev ${ }^{10,1}$, Yu. Lemeshev ${ }^{1}$, V. Lenok ${ }^{1}$, N. Lubsandorzhiev ${ }^{10}$, B. Lubsandorzhiev ${ }^{10,12}$, R. Mirgazov ${ }^{1}$, R. Mirzoyan ${ }^{13,1}$, R. Monkhoev ${ }^{1}$, R. Nachtigall ${ }^{9}$, E. Osipova ${ }^{1}$, A. Pakhorukov ${ }^{1}$, M. Panasyuk $^{10}$, L. Pankovi ${ }^{1}$, A. Petrukhin ${ }^{3}$, V. Poleschuk ${ }^{1}$, E. Popescu ${ }^{14}$, E. Popova ${ }^{10}$, A. Porelli ${ }^{5}$, E. Postnikov ${ }^{10}$, V. Prosin ${ }^{10}$, V. Ptuskin ${ }^{15}$, A. Pushnin ${ }^{1}$, E. Rjabov ${ }^{1}$, G. Rubtsov ${ }^{12}$, B. Sabirov ${ }^{4}$, Y. Sagan ${ }^{4}$, V. Samoliga ${ }^{1}$, Yu. Semeney ${ }^{1}$, A. Sidorenkov ${ }^{12}$, A. Silaev ${ }^{10}$, A. Silaev(junior) ${ }^{10}$, A. Skurikhin ${ }^{10}$, V. Slunecka ${ }^{4}$, A. Sokolov $^{7,11}$, C. Spiering ${ }^{5}$, L. Sveshnikova ${ }^{10}$, V. Tabolenko ${ }^{1}$, B. Tarashansky ${ }^{1}$, A. Tkachenko ${ }^{4}$, L. Tkachev ${ }^{4,8}$, M. Tluczykont ${ }^{9}$, R. Wischnewski ${ }^{5}$, I. Yashin ${ }^{3}$, A. Zagorodnikov ${ }^{1}$, V. Zurbanov ${ }^{1}$

${ }^{1}$ Institute of Applied Physics ISU, Irkutsk, Russia, ${ }^{2}$ Irkutsk National Research Technical University, Irkutsk, Russia, ${ }^{3}$ National Research Nuclear University MEPhI (Moscow Engineering Physics Institute), Moscow, Russia, ${ }^{4}$ JINR, Dubna, Russia, ${ }^{5}$ DESY, Zeuthen, Germany, ${ }^{6}$ Dipartimento di Fisica Generale Universiteta di Torino and INFN, Torino, Italy, ${ }^{7}$ Novosibirsk State University, NSU, Novosibirsk, Russia, ${ }^{8}$ Dubna State University, Dubna, Russia, ${ }^{9}$ Institute for Experimental Physics, University of Hamburg, Germany, ${ }^{10}$ Skobeltsyn Institute of Nuclear Physics MSU, Moscow, Russia, ${ }^{11}$ Budker Institute of Nuclear Physics SB RAS, Novosibirsk, Russia, ${ }^{12}$ Institute for Nuclear Research of RAN, Moscow, Russia,

${ }^{13}$ Max-Planck-Institute for Physics, Munich, Germany, ${ }^{14}$ ISS, Bucharest, Romania, ${ }^{15}$ IZMIRAN, Moscow, Russia

E-mail: sidney28@yandex.ru

In TAIGA (Tunka Advanced Instrument for cosmic ray physics and Gamma-ray Astronomy) detector we are commissioning the first Imaging Atmospheric Cherenkov Telescope (IACT). The telescope drive system comprises a motor controller and two stepper motors for axis control. For the pointing calibration of the telescope a CCD-camera is installed on the reflector dish of the telescope. The position of the CCD-camera allows us capturing both the Cherenkov camera and the observed source. In this report we describe the telescope positioning and the drive system software as well as the control of the CCD-camera data acquisition, archiving and processing system.

35th International Cosmic Ray Conference - ICRC2017

10-20 July, 2017

Bexco, Busan, Korea

* Speaker. 


\section{Introduction}

The TAIGA observatory [1] (Tunka Advanced Instrument for cosmic ray physics and Gamma Astronomy) is designed to solve the problems of the high energy gamma-ray astronomy and cosmic-ray physics. It is located in Tunka valley at a distance of $50 \mathrm{~km}$ from Lake Baikal. The TAIGA observatory is an unique complex ground-based system for detecting extensive air showers (EAS) produced by high-energy gamma-quanta and cosmic-ray particles. TAIGA consists of various types of detectors [1,2], including wide-angle detectors of Cherenkov radiation (Tunka-133 and TAIGA-HiSCORE [3, 4]), radio-antennas (Tunka-Rex [5]), detectors of electrons and muons of EAS (Tunka-GRANDE [6, 7]) as well as Image Atmospheric Cherenkov Telescopes TAIGAIACT.

One of the key ideas for the development of gamma astronomy with TAIGA detectors is the joint operation of the TAIGA-HiSCORE detectors and the array of atmospheric Cherenkov telescopes TAIGA-IACT $[8,9]$. The TAIGA-HiSCORE is array of wide-angle Cherenkov detectors currently consists of 28 stations distributed over an area of $0.25 \mathrm{~km}^{2}$ [3]. In December 2016, the first TAIGA-IACT telescope was installed in the Tunka valley. By 2019, it is planned to install 2 more IACT telescopes distributed at a distance of about $600 \mathrm{~m}$ from each other [2].

This article describes the telescope hardware as well as the tools used for software development. As the framework for the control system development a set of software tools EPICS was chosen. An algorithm for processing of images taken from a CCD-camera mounted on a telescope dish and intended for calibration is also described. The results of measuring of the deformation of the telescope construction are presented, as well as the results of the first tracking operations.

\section{The TAIGA-IACT telescope}

The telescope TAIGA-IACT (see Figure 1) has an alt-azimuth mount and a mirror in the DavisCotton design consisting of 34 segments, each is of $60 \mathrm{~cm}$ a diameter. The full diameter of the mirror is $4.3 \mathrm{~m}$ and the total area of the reflecting surface is about $10 \mathrm{~m}^{2}$. The Cherenkov camera of the telescope image consists of 547 photomultiplier tubes (PMTs) and has a field of view of about $10 x 10$ degrees. The angular resolution of the Cherenkov camera is 0.36 degrees per pixel[10]. On the camera of the atmospheric Cherenkov telescope 8 LEDs of artificial light are located along the perimeter. These LEDs are used to determine the position of the Cherenkov camera.

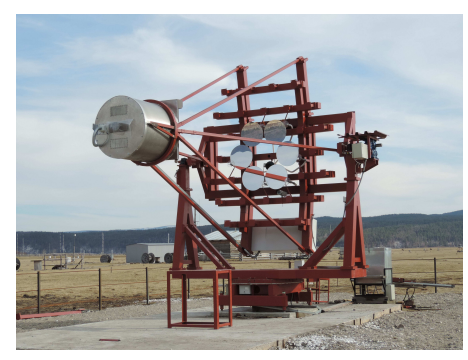

Figure 1: The TAIGA-IACT telescope located in the Tunka valley. The telescope is on the parking position and directed to the north. On the 2016-2017 data taking season only 6 of the 34 reflector segments is installed. 
To perform the calibration measurements and determine the accurate position of the telescope in the sky a CCD-camera Prosilica GC1380 was installed on a dish of the telescope near the mirrors. It was installed in such a way that this position allows us capturing on the one image the sky whith observed source as well as the telescope camera with the LEDs (see Figure 2). This makes it possible to determine the reference to the celestial coordinate system and the position of the Cherenkov camera simultaneously. To maintain operation conditions the CCD-camera is placed in a sealed casing WizeBox SVS32P (IP66).
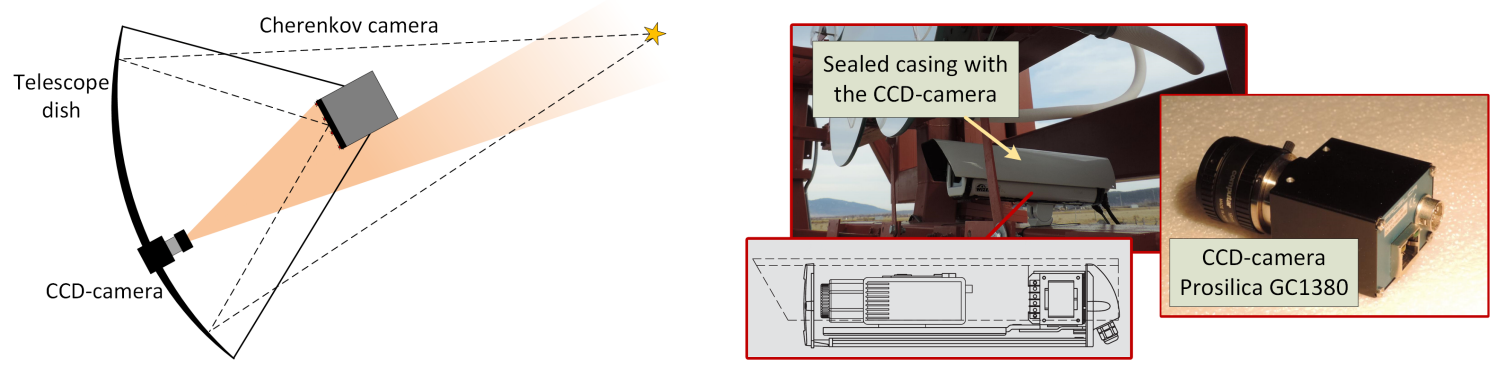

Figure 2: Left: CCD-camera field of view that allow capturing both the Cherenkov camera and the observed source. Right: CCD-camera Prosilica GC1380 with a sealed casing WizeBox SVS32P is installed on a dish.

The CCD-camera has a resolution of $1360 \times 1024$ pixels and contains 12-bit ADC. The camera can run up to 20 frames per second, and exposure time can be set from microseconds to one minute. On the CCD-camera a Computar 16mm Camera Lense 1:1.4 2/3" 04K is installed that gives the field of view of $30.8 \times 23.4$ degrees and the angular resolution of $82 \operatorname{arcsec}(22.8$ mdegree). The camera is powered by a power supply unit installed inside the sealed casing and connected to an AC 220V. Remote access to the camera for control and data acquisition is avalable over Ethernet network.

To control the movement of the telescope hybrid stepper motors Phytron ZSH107/4.200.12,5 are installed on each axis in a heated box. Each motor performs one revolution in 200 full motor steps and is connected to the axles through 2 gearboxes: the first with a gear ratio of 40 , and the second with a gear ratio of 50. Thus, the telescope turns 360 degrees in 400000 full steps. Also each axis of the telescope is equipped with 2 limit switches, which in case the telescope leaves the permissible angles send a signal to the drive system controller. Stepper motors and limit switches as well as encoders are connected to the PhyMOTION control unit, a modular stepper motor control system that, among other function, supports micro step operation mode up to $1 / 512$ of a full motor step that allows to perform smooth tracking. Remote access to the controller is available via Ethernet network.

\section{Software}

As framework for development of the TAIGA-IACT telescope pointing and control system software a set of software tools EPICS[11] is used. All necessary programs, libraries as well as EPICS base version 3.15.4 with required modules are installed on a computer running the operating system Ubuntu 14.04 LTS. This computer is located in the data acquisition center and in addition to the control system also includes data acquisition software. 
To work with the CCD-camera AreaDetector module is used, that provides driver and plugin system, which makes it possible to easily implement the on-the-fly image processing. Determination of the direction of the telescope in the celestial coordinates on-the-fly, in addition to monitoring, can be used to operate the drive system. Also the resulting images from CCD-camera are saved in FITS files along with the data from the drive system. These files can be used for further offline analysis.

The drive system is controlled using the appropriate drivers for the motor controller. To provide accurate telescope source tracking, each axis operates in velocity control mode. To calculate the position of the source in the coordinates of the azimuth and altitude angle software SOFA[12] (Standards of Fundamental Astronomy) is used, which allows performing complex transformations and take into calculation different corrections for accurate estimation.

For remote operation of the telescope a graphical user interface was developed using the EPICS Qt Framework. This allows to promptly display the necessary for the operator information in a convenient form and provide quality control over the process. For the possibility of supervision the telescope at a distance of about 30 meters an IP-camera was installed.

\section{The CCD-camera image processing}

To accurately determine the direction of the telescope in celestial coordinates using the CCDcamera images a number of calculations should be performed. Received images are divided into 2 region: the Cherenkov camera region and the sky region. From the first region the position of Cherenkov camera is determined and it is transformed to the sky region. Then Cherenkov camera position on the sky region is transformed to celestial coordinates using WCS parameters that are determined by Astrometry.net software. After that celestial coordinates are translated to azimuth and altitude angle which could be used by drive system. The data flow diagram shown in Figure 3 presents this process detailed.

To determine the position of the Cherenkov camera eight LEDs are used, each of which is equidistant from the center of the camera. The position of the LEDs is estimated as the center of gravity of their light distribution taking into calculation the pixels exceeding the established threshold above the background.

To mapping the obtained positions of the Cherenkov camera to the celestial region of the image in the pixel coordinates a transformation is used, that parameters are determined experimentally. Using the transformed camera center position, the direction of the telescope in the International Celestial Coordinate System ICRS (RA, Dec) is calculated, using the World Coordinate System (WCS) transformation parameters, obtained through astrometry.net [13, 14] software and index files based on the astronomical catalogue Tycho-2 [15]. Only region with stars in field of view of the Cherenkov camera is used to determine the WCS parameters (see Figure 4).

The SOFA [12] software libraries are used to determine the azimuth and altitude angles (Az, Alt) of the source. In addition to the source right ascension and declination it also takes into calculation the position of the observer (telescope) and the time-stamp of the image taking. 


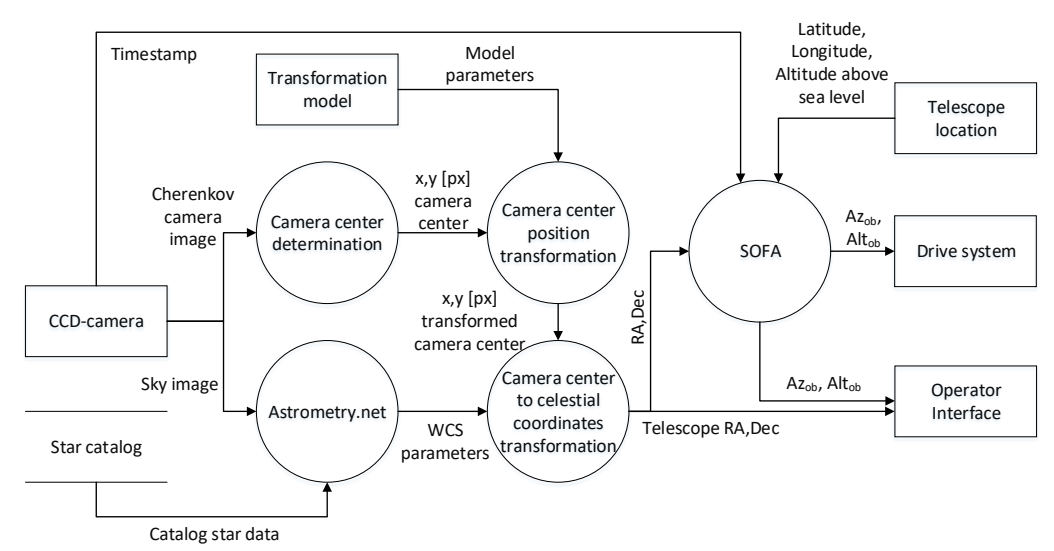

Figure 3: The data flow diagram that describes CCD-camera image processing and determination of the telescope direction in RA-Dec and Az-Alt coordinates. Received images from CCD-camera are divided into 2 region and are passed to the corresponding routines. Determined Cherenkov camera position is transformed to the sky region and then to celestial coordinetes using the WCS transformation parameters obtained by Astrometry.net software. Using SOFA library Alt-Az coordinates of telescope are calculated wich can be used by drive system.

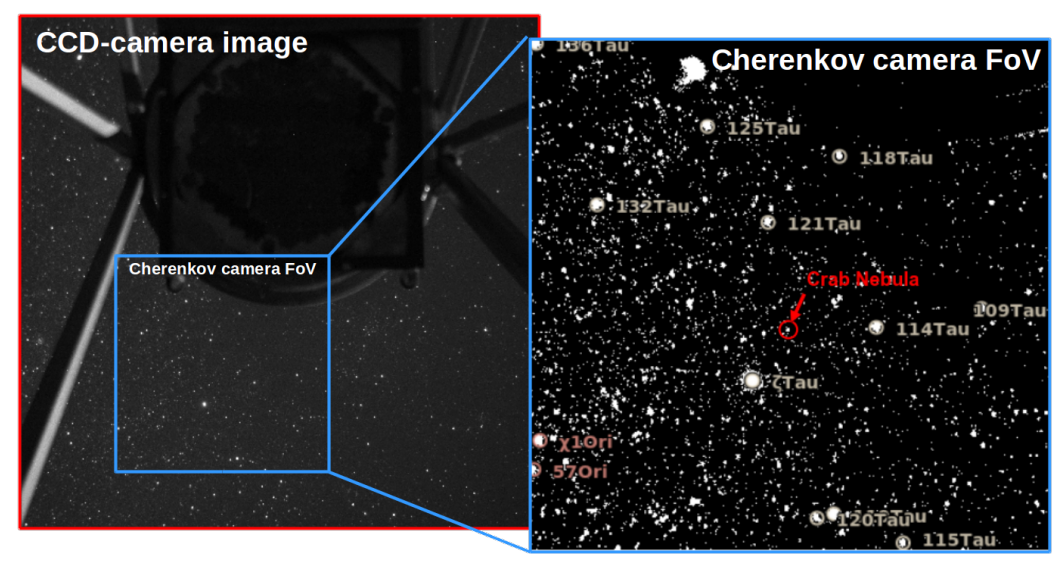

Figure 4: The CCD-camera field of view (left). For the Astrometry.net software CCD-image processing only region in field of view of the Cherenkov camera is used. In processed region (right) different sources as well as Crab Nebula can be seen.

\section{Deformation of the telescope construction}

For studing of telescope construction deformation an experiment was conducted, that based on the measurments of the position of the LEDs and the determination of the position of the Cherenkov camera. The result of this experiment are shown in Figure 5. During the experiment the telescope did not change its position along the azimuth axis and was directed to the north.

The telescope was raised up from a horizontal position to 72.5 degrees by altitude with 2.5 degree steps. At each step the telescope stopped and conducted a series of images from a CCDcamera. After reaching the upper position, the telescope was moved down to a horizontal position with measurements taken at each of 10 degrees step. As it can be seen maximum deviation from mean position of the Cherenkov camera does not exceed 0.02 degree. For accurate pointing and 


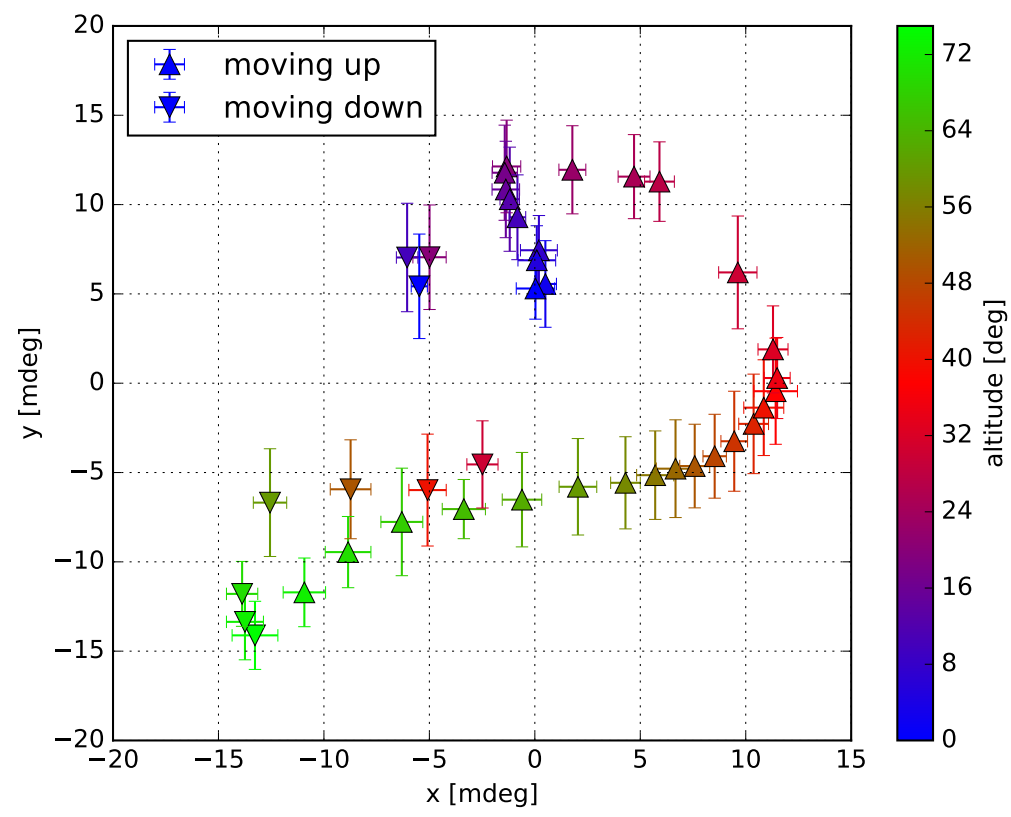

Figure 5: The position of the Cherenkov camera in a CCD image in angular degrees depends on telescope altitude. Mean value of Cherenkov camera position was taken as origin. The direction of telescope motion is shown as triangles directed up and down respectively. Color indicates an altitude.

tracking this effect should be taken into calculation.

\section{Test operations in tracking mode}

For the first test operations in the tracking mode in the absence of encoders on the axes feedback from the CCD-camera was implemented. These operations were conducted in clear, cloudless, windless nights. The resulting images from the CCD-camera were processed on the fly, that allowed calculating the azimuth and altitude position of the telescope. The position of the Cherenkov camera on images was used as a fixed value that means that the present result does not take into calculation the deformation of the construction and shows only the result of the drive system operation. The first tracking result is shown in Figure 6. In this operation the Vega star was chosen as target source and for 13 minutes 807 CCD-camera images were obtained and processed. The exposure time for each image was set to 1 second and gain level was set to $30 \mathrm{~dB}$.

A high deviation in telescope pointing is caused by a high dispersion of the azimuthal axis, which is evident from the distributions and the scatter plot. It is planned to fix this problem in the summer of 2017. Nevertheless, from the pointing error distribution, it can be seen that the tracking accuracy was higher than the CCD-camera resolution which is equal 22.8 mdegree. However, this approach with feedback to the CCD-camera is not planned to be used as the main in the future. The main approach that will be used in the following operations is the use of feedback from the shaft encoders and a pointing model. 

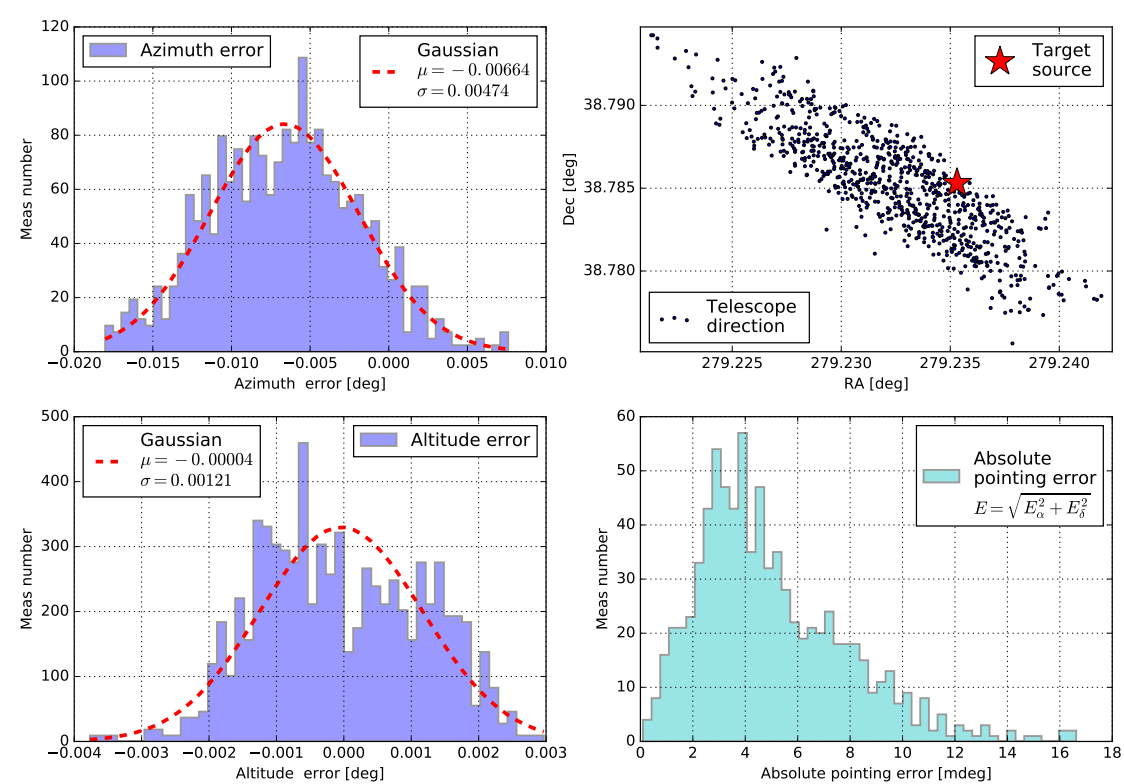

Figure 6: The tracking result with feedback from the CCD-camera. In the left histograms azimuth and altitude error distributions are prestnted. In the upper right corner scatter plot with telescope direction in RA and Dec coordinates and the target source is shown. Distributions of absolute pointing error calculated as the distance between the target and the measured position in the RA and Dec coordinates is shown in the lower right histogram.

\section{Conclusion}

The first image atmospheric Cherenkov telescope TAIGA-IACT was installed in the Tunka valley in December 2016. The Prosilica GC1380 CCD-camera is installed on the telescope for the pointing calibration and its position allows capturing both the Cherenkov camera and the observed source. A set of software tools EPICS was used to develop the control system for the drive system and the CCD-camera control. The image processing software allows to determine the telescope position on the fly. For test operations in the absence of shaft encoders, tracking of the source with feedback from the CCD-camera was implemented. After commissioning the first TAIGA-IACT telescope it will operate using the shaft encoders feedback and a pointing model.

\section{Acknowledgements}

This work was supported by the Russian Federation Ministry of Education and Science (projects 14.B25.31.0010, 14.593.21.0005, 3.10131.2017/NM, 2017-14-595-0001-003, No3.9678.2017/8.9, No3.904.2017/4.6, 3.6787.2017/7.8, 1.6790.2017/7.8), the Russian Foundation for Basic Research (Grants 16-02-00738, 16-32-00329, 17-02-00905, 16-29-13035), the grant 15-12-20022 of the Russian Science Foundation (section 3 and 6), the German Helmholtz Association (grant HRJRG303), the DFG (grant TL 51-3) and the European Union's Horizon 2020 programme under grant agreement No. 653477. 


\section{References}

[1] N.Budnev et al. (TAIGA Collaboration) Nucl. Instr. Meth. Phys. Res. A 845 (2017) 330-333. http://iopscience.iop.org/article/10.1088/1742-6596/718/5/052006/pdf

[2] N.Budnev et al. (TAIGA Collaboration) The TAIGA observatory - a hybrid detector complex for high energy gamma-ray astronomy and cosmic ray physics in the Tunka valley. These conference proceedings

[3] M.Tluczykont et al. (TAIGA Collaboration) Status of the HiSCORE detector component of the TAIGA experiment. These conference proceedings

[4] O.Gress et al. (TAIGA Collaboration) Nucl. Instr. Meth. Phys. Res. A 845 (2017) 367-372.

[5] P.A. Bezyazeekov et al. (Tunka-Rex Collaboration) Nucl. Instr. and Meth. A 802 (2015) 89-96, doi:10.1016/j.nima.2015.08.061

[6] N.Budnev et al. The Tunka-Grande experiment - present status and perspectives. These conference proceedings

[7] R. Monhoev et al. (Tunka Collaboration), Bull. Russian Academy of Sciences: Physics, 81, 504 (2017)

[8] M.Tluczykont et al. Towards gamma-ray astronomy with timing arrays. Journal of Physics: Conference Series. Vol. 632. No. 1. IOP Publishing, 2015 http://iopscience.iop.org/article/10.1088/1742-6596/632/1/012042/pdf

[9] L. Sveshnikova et al. ( TAIGA Collaboration) Commissioning the joint operation of the wide angletiming HiSCORE Cherenkov array with the first IACT of the TAIGA experiment. These conference proceedings

[10] I.Yashin et al. The Taiga project. Journal of Physics: Conference Series. Vol. 675. No. 3. IOP Publishing, 2016

[11] http://www.aps.anl.gov/epics/

[12] http://www.iausofa.org/

[13] http://www.astrometry.net/

[14] Lang, D., Hogg, D.W., Mierle, K., Blanton, M., Roweis, S., 2010, Astrometry.net: Blind astrometric calibration of arbitrary astronomical images, The Astronomical Journal 139, 1782-1800.

[15] E.Hog et al. The Tycho- 2 catalogue of the 2.5 million brightest stars. Astronomy and Astrophysics 355 (2000): L27-L30.

[16] L.Sveshnikova et al (TAIGA Collaboration) The search for gamma emission above $50 \mathrm{TeV}$ from Crab Nebula in the TAIGA observatory. These conference proceedings

[17] A.Porelli e $\mathrm{t}$ al (TAIGA Collaboration) TAIGA-HiSCORE observation of the CATS-Lidar on the ISS as fast moving point source. These conference proceedings

[18] N. Lubsandorzhiev et al ( TAIGA Collaboration) Camera of the first TAIGA-IACT: construction and calibration. These conference proceedings

[19] A.Porelli,R.Wischnewski,M.Tluczykont A hybrid time calibration method for EAS ground-based timing arrays. These conference proceedings 